\title{
Philosophiques
}

\section{Commentaire sur Emmanuel Barot : Lautman}

\section{Pierre Cassou-Noguès}

Volume 37, numéro 1, printemps 2010

Albert Lautman, philosophe des mathématiques

URI : https://id.erudit.org/iderudit/039720ar

DOI : https://doi.org/10.7202/039720ar

Aller au sommaire du numéro

Éditeur(s)

Société de philosophie du Québec

ISSN

0316-2923 (imprimé)

1492-1391 (numérique)

Découvrir la revue

Citer ce document

Cassou-Noguès, P. (2010). Commentaire sur Emmanuel Barot : Lautman.

Philosophiques, 37(1), 197-200. https://doi.org/10.7202/039720ar d'utilisation que vous pouvez consulter en ligne.

https://apropos.erudit.org/fr/usagers/politique-dutilisation/ 


\title{
Commentaire sur Emmanuel Barot: Lautman
}

\author{
PIERRE CASSOU-NOGUÈS \\ CNRS et Université Lille III
}

Les noms des philosophes de cette génération semblent aller, dans notre imaginaire, par paire: Sartre et Merleau-Ponty, ou Cavaillès et Lautman. Cependant, alors que les œuvres de Sartre et de Merleau-Ponty font chacune l'objet d'études indépendantes, il reste difficile de parler de Lautman sans évoquer Cavaillès. De quelques années son cadet - Cavaillès est né en 1903, Lautman en 1908 —, élève de Cavaillès à l'ENS — la même année que Merleau-Ponty —, se plongeant à la suite de Cavaillès dans les mathématiques contemporaines, résistant pendant la guerre, fusillé quelques mois après Cavaillès, Lautman est par ce cheminement comme par l'amitié qui les liait, très proche de Cavaillès. Leurs œuvres partagent aussi une référence aux mathématiques de leur temps et un fort héritage brunschvicgien. Cela dit, il faut bien reconnaître que le travail de Lautman ouvre une autre perspective, qui est différente de celle de Cavaillès ou n'en est pas, comme on voudrait parfois le croire, un double à la fois un peu pâle et un peu obscur. Or, malgré le volume édité par F. Zalamea en 2006, l'œuvre de Lautman reste méconnue. L'ouvrage d'Emmanuel Barot est la première monographie qui lui soit consacrée. Et il faut saluer l'excellente analyse, aussi instructive que stimulante, que donne E. Barot. On y trouve une présentation claire des principales sources de Lautman, y compris des grandes notions mathématiques qu'utilise Lautman, et un examen précis des problèmes que Lautman a rencontrés et sur lequel il a sans doute buté jusqu'à la fin de cette œuvre interrompue par une mort prématurée.

Le premier chapitre discute des trois « cadres» de la pensée de Lautman: le développement des mathématiques au XIX ${ }^{e}$ siècle jusqu'à la controverse sur le problème des fondements dans les années vingt; la métamathématique de Hilbert, que E. Barot isole en raison de l'importance que lui donne Lautman et de l'interprétation singulière qu'il en propose; la tradition épistémologique en France, de Brunschvicg, Bachelard ou Cavaillès, dans laquelle s'inscrit également Lautman.

Le deuxième chapitre, "Du réel en mathématique », retrace dans ses grandes lignes l'épistémologie des mathématiques de Lautman à partir de la distinction entre le niveau proprement mathématique, le niveau des théories mathématiques, et le niveau idéal, le niveau des Idées qui animent les théories mathématiques et leur donnent leur objectivité. Il serait inutile, dans ce volume consacré tout entier à la pensée de Lautman, de tenter d'esquisser en quelques lignes son épistémologie des mathématiques. Il faut souligner cependant que E. Barot en profite pour introduire de façon très pédagogique 
aux notions mathématiques auxquelles se réfère Lautman dans ses principaux exemples.

Le troisième chapitre, "La différence ontologique face à l'histoire", est consacré au grand problème de la pensée de Lautman, celui du rapport entre les Idées et les théories mathématiques. Comment penser le rapport entre ses deux niveaux, l'un qui est historique, celui des théories mathématiques, et l'autre, celui des idées, qui n'a pas à proprement parler d'histoire? Comment penser la "procession", l'incarnation pour ainsi dire des Idées dans les théories mathématiques? E. Barot distingue trois modèles, montrant à chaque fois leur inadéquation à la pensée de Lautman ou la façon dont Lautman déforme ces modèles antérieurs pour tenter de fixer ce rapport des Idées aux mathématiques. Le premier modèle est platonicien. Le deuxième modèle, qui s'appuie sur quelques références de Lautman au philosophe allemand, est heideggerien. Le troisième modèle, que propose E. Barot, est hegelien. Il faut dire que Lautman lui-même ne se réfère jamais à Hegel. Mais le point de départ d'E. Barot - qui s'inscrit dans une thèse plus large sur un hegelianisme inavoué de la tradition épistémologique en France - est que le développement des Idées dans les théories mathématiques relève d'une dialectique que l'on peut comparer à celle de Hegel: «du fait que ces Idées sont des schémas de liaisons possibles entre notions contraires, la dynamique de leur réalisation est bien une antithétique de style hégélien " (p. 146, E. Barot souligne). Les Idées sont formées de couples de notions opposées, le discret et le continu, le local et le global, et les théories mathématiques ont bien pour but de lier, en un sens, ces notions opposées. Cela dit, E. Barot doit immédiatement reconnaître, d'une part, que le développement des théories mathématiques les unes à la suite des autres ne relève pas de la seule négativité, comme c'est le cas dans la dialectique de Hegel, et d'autre part, que les oppositions, qui se marquent dans l'épistémologie de Lautman au sein des Idées, ne sont pas dépassées, annulées, dans les théories mathématiques, comme elles le sont dans la dialectique de Hegel. Si l'on veut, l'arithmétisation de l'analyse donne une version de l'opposition entre le discret et le continu, une façon de lier ces notions idéales, mais ne les dépasse pas pour les intégrer dans une troisième notion - comme l'être et le néant se dépasse dans la dialectique hégélienne dans le devenir - qui pourrait être à son tour niée pour former une nouvelle opposition à son tour dépassée, et ainsi de suite jusqu'au savoir absolu. Les oppositions idéales dans l'épistémologie de Lautman ne font pas l'objet d'une telle "sursomption ». Ce troisième modèle, pour intéressant qu'il soit, est finalement un échec: «Il n'y a pas d'Aufhebung chez Lautman, ni téléologie, ni finalisme de l'Idée, ni tension vers un savoir absolu réunifié» (p. 148). La nature de la «différence ontologique », si elle est éclairée par ces trois modèles, reste ce qu'elle est dans les textes de Lautman: un problème.

Le quatrième chapitre aborde les derniers textes sur la physique. La conclusion retrace les grandes interprétations à laquelle l'œuvre de Lautman 
a donné lieu. E. Barot distingue deux réceptions mathématiques: avec Bourbaki et l'interprétation de l'Idée comme "structure»; dans la lecture de F. Zalamea qui rapproche la pensée de Lautman de la théorie des catégories. La brève généalogie des réceptions philosophiques passe par la position de Deleuze dans Différence et répétition, la perspective phénoménologique de J. Petitot et de J.-M. Salanskis, et le platonisme de A. Badiou. Je dois dire que, dans l'analyse de ces réceptions, E. Barot ne donne pas à mes yeux assez de place à la lecture que fait Deleuze de Lautman, dans Différence et répétition. Historiquement, celle-ci a été tout à fait importante aussi bien dans l'économie de la pensée de Deleuze que pour la réception de l'œuvre de Lautman. En fait, Deleuze a donné la principale, sinon la seule, lecture de Lautman chez les philosophes entre les années quarante et les années quatrevingt.

Mais je voudrais surtout revenir sur la «différence ontologique» et le problème de définir le rapport entre le niveau des Idées et celui du développement mathématique: comment ce qui n'a pas à proprement parler d'histoire peut-il venir diriger, ou donner son objectivité, à un développement qui est historique? On pourrait montrer, je crois, que ce problème n'est pas résolu par Lautman, que le philosophe y revient toujours à nouveau pour lui proposer des solutions différentes et qui restent insatisfaisantes.

Or, en se plaçant sans doute à une certaine distance, le problème de Lautman semble trouver un écho de ce qu'il est convenu d'appeler le dilemme de Benacerraf. Pour le formuler rapidement, dans l'article de 1973, "Mathematical Truth ", P. Benacerraf montre que la philosophie des mathématiques est confrontée à une alternative: ou bien elle met l'accent sur la notion de vérité, elle est alors conduite à poser des objets idéaux, auxquels les énoncés mathématiques se réfèrent, mais elle ne peut alors rendre compte de notre connaissance de ces objets idéaux; ou bien elle met l'accent sur la nécessité, disons épistémologique, de rendre compte de la connaissance mathématique, elle est alors conduite à une sorte de formalisme, qui ne permet pas d'obtenir une notion satisfaisante de vérité en mathématiques. Sans doute, les Idées de Lautman ne se confondent pas avec les objets idéaux auxquels pense P. Benacerraf. La réalité idéale de la théorie des ensembles, pour Lautman, serait dans les notions de continu et du discret plutôt que dans un univers d'ensembles qu'elle décrirait et qui rendrait «vrais » ces énoncés. Les Idées de Lautman ne représentent pas des modèles pour les théories mathématiques. D'autre part, lorsque $\mathrm{P}$. Benacerraf pose comme problème notre connaissance des objets mathématiques - dans l'hypothèse où l'on accepte de tels objets idéaux —, il l'entend dans le cadre d'une théorie causale, selon laquelle je ne peux connaître un objet, ou un état de chose, que lorsque celui-ci exerce une influence causale qui s'étend jusqu'à moi: je ne peux percevoir et ne peux connaître que la neige tombe que si le fait que la neige tombe a une influence causale sur moi. Et le problème alors est qu'un objet idéal hors de l'espace et du temps ne semble pas pouvoir exercer d'influence 
causale sur un être qui s'inscrit dans l'espace et dans le temps. Lautman n'accepterait sans doute pas une telle théorie de la connaissance. Néanmoins, et avec ces différences, il est confronté à un dilemme qui reste semblable à celui que met en évidence Benacerraf. D'un côté, Lautman est convaincu que des épistémologies comme celles de Cavaillès sont insuffisantes: il faut inscrire dans l'expérience mathématique la référence à une objectivité idéale et extérieure à l'expérience mathématique proprement dite. Mais, d'un autre côté, dès lors que l'on pose une réalité idéale, qui n'a pas la temporalité des théories mathématiques, le rapport des théories mathématiques à cette réalité idéale et la façon dont nous développons cette réalité idéale dans des théories mathématiques apparaissent incompréhensibles, et ni la référence à Platon, ni celle à Heidegger, ni un éventuel écho hegelien ne suffisent à résoudre de façon satisfaisante ce problème. Il faudrait donc ou bien mettre l'accent sur le développement des théories mathématiques - et en ce sens la connaissance mathématique, en excluant toute référence à une objectivité extérieure, ce qui conduit à une épistémologie comme celle de Cavaillès qui pour Lautman est insuffisante - ou bien accepter la référence à une objectivité extérieure au risque de perdre alors le moyen de rendre compte de la connaissance mathématique à partir de cette réalité idéale. C'est, à une certaine distance bien sûr, et surtout traduit dans les termes d'une tout autre tradition philosophique, un dilemme analogue à celui de P. Benacerraf.

Lautman me semble participer à une sorte de renouveau du platonisme qui marque en profondeur la philosophie des mathématiques de son époque, de Russell à Gödel, disons, mais que l'on retrouverait également à l'extérieur de la philosophie des mathématiques. Je pense par exemple aux «idées sensibles » que Proust met en évidence - cet élément dans l'expérience de la madeleine, qui semble traverser le temps pour ramener en un seul bloc l'enfance du narrateur à Combray - idées sensibles qu'a reprises MerleauPonty et qui se lient dans le virtuel, tel que le décrit Deleuze, aux Idées de Lautman. C'est peut-être alors dans un contexte plus large qu'il s'agirait de replacer le platonisme de Lautman et les problèmes que le philosophe rencontre pour les éclairer, même dans ce que ceux-ci peuvent avoir d'insolubles au regard de nos catégories usuelles. Mais il ne fait pas de doute qu'il faut d'abord lire Lautman dans la perspective des traditions épistémologiques et mathématiques qui sont les siennes, et dans lesquels son platonisme prend sa spécificité. Et, de ce point de vue, je le répète, l'étude de E. Barot est excellente et donne une introduction aussi intéressante que pédagogique aux notions dont part Lautman et à ses principales thèses. 